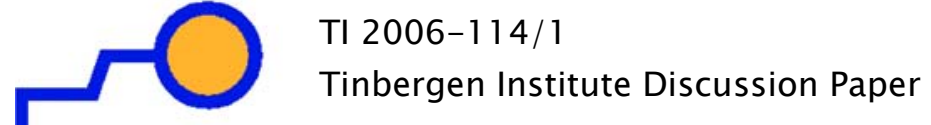 Distributing Dividends in Games with Ordered Players
}

\author{
René van den Brink ${ }^{7}$ \\ Gerard van der Laan' \\ Valeri Vasil'ev²
}

1 Vrije Universiteit Amsterdam, and Tinbergen Institute;

2 Sobolev Institute of Mathematics, Novosibirsk, Russia. 


\section{Tinbergen Institute}

The Tinbergen Institute is the institute for economic research of the Erasmus Universiteit Rotterdam, Universiteit van Amsterdam, and Vrije Universiteit Amsterdam.

Tinbergen Institute Amsterdam

Roetersstraat 31

1018 WB Amsterdam

The Netherlands

Tel.: $\quad+31(0) 205513500$

Fax: $\quad+31(0) 205513555$

Tinbergen Institute Rotterdam

Burg. Oudlaan 50

3062 PA Rotterdam

The Netherlands

Tel.: $\quad+31(0) 104088900$

Fax: $\quad+31(0) 104089031$

Most TI discussion papers can be downloaded at http:/ /www.tinbergen.nl. 


\title{
Distributing dividends in games with ordered players ${ }^{1}$
}

\author{
René van den Brink ${ }^{2}$ Gerard van der Laan ${ }^{3}$ Valeri Vasil'ev ${ }^{4}$
}

December 15, 2006

${ }^{1}$ This research has been done while the third author was visiting the Tinbergen Institute, Free University Amsterdam, on NWO-grant 047.017.017 within the framework of Dutch-Russian cooperation.

2J.R. van den Brink, Department of Econometrics and Tinbergen Institute, Free University, De Boelelaan 1105, 1081 HV Amsterdam, The Netherlands. E-mail: jrbrink@feweb.vu.nl

${ }^{3}$ G. van der Laan, Department of Econometrics and Tinbergen Institute, Free University, De Boelelaan 1105, $1081 \mathrm{HV}$ Amsterdam, The Netherlands. E-mail: glaan@feweb.vu.nl

${ }^{4}$ V.A. Vasil'ev, Sobolev Institute of Mathematics, Prosp. Koptyuga 4, 630090 Novosibirsk, Russia, E-mail: vasilev@math.nsc.ru 


\begin{abstract}
A situation in which a finite set of players can obtain certain payoffs by cooperation can be described by a cooperative game with transferable utility, or simply a TU-game. A solution for TU-games assigns a set of payoff vectors to every TU-game. Some solutions that are based on distributing dividends are the Shapley value (being the single-valued solution distributing the dividends equally among the players in the corresponding coalitions) and the Selectope or Harsanyi set (being the set-valued solution that contains all possible distributions of the dividends among the players in the corresponding coalitions).

In this paper we assume the players to be hierarchically ordered. We modify the concept of Harsanyi set to this context by taking into account this hierarchical order when distributing the dividends of the game. We show that the resulting new solution concept for games with ordered players, called the Restricted Harsanyi set, is fully characterized by a collection of seven logically independent properties. We also discuss an alternative modification of the Harsanyi set and a solution concept resulting from adapting the concept of Selectope to games with ordered players. Some applications show the usefulness of the Restricted Harsanyi set.
\end{abstract}

Keywords: TU-game, Harsanyi dividends, Shapley value, Harsanyi set, Selectope, digraph.

AMS subject classification: 91A12 (Cooperative games), 5C20 (Directed graphs) JEL code: C71 (Cooperative games) 


\section{Introduction}

A situation in which a finite set of players can obtain certain payoffs by cooperation can be described by a cooperative game with transferable utility, or simply a TU-game. A solution for TU-games assigns a set of payoff vectors (possibly empty or consisting of a unique element) to every $T U$-game. In a payoff vector provided by a solution, the payoff assigned to a particular player depends on the payoffs that can be obtained by any coalition of players. The most well-known single-valued solution is the Shapley value (Shapley, 1953), the most well-known set-valued solution is the Core (Gillies, 1953).

In this paper we assume that the players in a TU-game are part of some hierarchical structure that is represented by a directed graph. In such games with ordered players the payoff assigned to a player may depend on both the worths of the coalitions and the position of the player in the graph. We introduce a solution for such games with ordered players that is based on distributing the Harsanyi dividends (see Harsanyi, 1959) of a game in the spirit of the Harsanyi set or Selectope. The dividend of a singleton is equal to its worth while, recursively, the dividends of all other coalitions are defined as their worth minus the dividends of all proper subcoalitions. In this sense the dividend of a coalition might be considered as the earnings of cooperation of the coalition that was not yet realized by its proper subcoalitions. The Harsanyi set, see Vasil'ev $(1978,1981)$ and Vasil'ev and van

der Laan (2002),assigns to every TU-game all Harsanyi payoff vectors, being those payoff vectors that are obtained by distributing every dividend in any possible way among the players in the corresponding coalition. An alternative definition of this set is given by the Selectope of a TU-game, see Hammer et. al. (1977) and Derks et. al. (2000), defined as the convex hull of all selectope vectors, where the selectope vectors are those vectors where every dividend is fully assigned to one player of the corresponding coalition.

For games with ordered players we modify the Harsanyi set by requiring that any dividend is distributed in such a way that a player that is dominated within the coalition gets a share in this dividend that is at most equal to the share in this dividend assigned to a player by whom he is dominated. We refer to this set as the Restricted Harsanyi set and refer to the corresponding solution as the Restricted Harsanyi set solution. The Restricted Harsanyi set generalizes both the Shapley value and the Harsanyi set for standard TUgames. In particular, for any directed graph the Shapley value of the TU-game (without ordered players) belongs to the Restricted Harsanyi set, and it is the unique element of this set if the digraph is complete, i.e., when each player $i$ dominates each other player $j$. On the other hand, for any directed graph the Restricted Harsanyi set is a subset of the Harsanyi set (of the game without ordered players), and it equals this Harsanyi set in case the graph is empty.

We provide a full characterization of the Restricted Harsanyi set solution on the class 
of games with ordered players as the unique nonempty solution that satisfies a collection of seven logically independent axioms. Besides the well-known efficiency, null player property, disjoint additivity and sign-preservation properties, it is characterized by convexity and two properties reflecting the hierarchical aspect of games with ordered players, namely an inferior player property and a consistency property. We also show that any solution satisfying all axioms except the consistency property assigns to every game with ordered players a subset of the Restricted Harsanyi set.

Further, besides a second modification of the Harsanyi set characterized by alternative inferior player and consistency properties, we also discuss shortly how to modify the concept of Selectope to games with ordered players. Although for standard TU-games the Selectope and Harsanyi set are equivalent, it appears that for games with ordered players their modifications give different solutions. In fact, the Restricted Selectope solution assigns to every game with ordered players a (possibly empty) subset of the (always nonempty) Restricted Harsanyi set.

The paper is organized as follows. In Section 2 we discuss some preliminaries on TU-games and directed graphs. In Section 3 we introduce the Restricted Harsanyi set for games with ordered players and show some of its properties. In Section 4 we characterize the Restricted Harsanyi set solution by seven logically independent axioms. In Section 5 we provide an alternative modification of the Harsanyi set and we discuss the concept of Restricted Selectope. An application is given in Section 6, while final remarks are made in Section 7.

\section{Preliminaries}

\subsection{TU-games}

A cooperative game with transferable utility, or simply a TU-game, is a pair $(N, v)$, where $N=\{1, \ldots, n\}$ is a finite set of $n$ players, and $v: 2^{N} \rightarrow \mathbb{R}$ is a characteristic function on $N$ such that $v(\emptyset)=0$. For any coalition $S \subseteq N, v(S)$ is the worth of coalition $S$, i.e., the members of coalition $S$ can obtain a total payoff of $v(S)$ by agreeing to cooperate. Since we take the player set $N$ to be fixed, we represent a TU-game by its characteristic function $v$. We denote the collection of all TU-games on $N$ by $\mathcal{G}^{N}$. A special class of TU-games are unanimity games. For $T \subseteq N, T \neq \emptyset$, the unanimity game $u_{T}$ on $N$ is given by $u_{T}(S)=1$ if $T \subseteq S$, and $u_{T}(S)=0$ otherwise. Writing $v$ as a $\left(2^{n}-1\right)$-dimensional vector with the worths of the $2^{n}-1$ nonempty coalitions as its components, it is well-known that every TU-game $v$ is a unique linear combination of unanimity games, i.e., there exist uniquely determined weights $\Delta_{v}(T) \in \mathbb{R}$ such that $v=\sum_{T \in 2^{N} \backslash\{\emptyset\}} \Delta_{v}(T) u_{T}$. These weights usually are called the (Harsanyi) dividends. Recursively, solving $v(S)=\sum_{T \subseteq S} \Delta_{v}(T)$ on the 
number of players starting from $\Delta_{v}(\{i\})=v(\{i\})$ for the single player coalitions yields $\Delta_{v}(T)=v(T)-\sum_{S \subset T} \Delta_{v}(S)=\sum_{S \subseteq T}(-1)^{|T|-|S|} v(S)$ for $T \subseteq N$ (see Harsanyi (1959)). A TU-game $v$ is almost positive if $\Delta_{v}(S) \geq 0$ for all $S \subseteq N$ with $|S| \geq 2$, and it is totally positive if also $\Delta_{v}(\{i\})=v(\{i\}) \geq 0$ for all $i \in N$. Every almost positive game $v$ is a convex game, i.e. $v(S \cup T)+v(S \cap T) \geq v(S)+v(T)$ for all $S, T \subseteq N$.

A set-valued solution $F$ on $\mathcal{G}^{N}$ assigns a set $F(v) \subset \mathbb{R}^{n}$ of payoff vectors to every TUgame $v \in \mathcal{G}^{N}$. A single-valued solution $F$ on $\mathcal{G}^{N}$ assigns precisely one vector $F(v) \in \mathbb{R}^{n}$ to every $v \in \mathcal{G}^{N}$. Examples of set-valued solutions are the Core and the Selectope or Harsanyi set. The Core of TU-game $v \in \mathcal{G}^{N}$ is the set of all efficient payoff vectors that are stable in the sense that no coalition can do better by separating, i.e., Core $(v)=\left\{x \in \mathbb{R}^{n} \mid \sum_{i \in N} x_{i}=\right.$ $v(N)$ and $\sum_{i \in S} x_{i} \geq v(S)$ for all $\left.S \subseteq N\right\}$. As known, the Core of a game can be empty ${ }^{1}$. A set-valued solution $F$ on $\mathcal{G}^{N}$ is said to be nonempty when $F(v) \neq \emptyset$ for any $v \in \mathcal{G}^{N}$.

The Selectope or Harsanyi set is nonempty for every TU-game. A selector chooses for every coalition a particular player in the coalition to whom to assign the dividend of that coalition, i.e., a selector is a function $\alpha: 2^{N} \backslash\{\emptyset\} \rightarrow N$ such that $\alpha(T) \in T$ for all $T \subseteq N$. The selectope vector corresponding to selector $\alpha$ and game $v \in \mathcal{G}^{N}$ is the vector $s^{\alpha}(v) \in \mathbb{R}^{n}$ given by $s_{i}^{\alpha}(v)=\sum_{\substack{T \in 2^{N} \backslash\{\emptyset\} \\ \alpha(T)=i}} \Delta_{v}(T)$. The Selectope $S(v)$ of $v$ then is the convex hull of all selectope vectors (see Hammer et. al. (1977) and Derks et. al. (2000), i.e.,

$$
S(v)=\operatorname{Conv}\left(\left\{s^{\alpha}(v) \mid \alpha \in \mathcal{A}^{N}\right\}\right),
$$

with $\mathcal{A}^{N}=\left\{\alpha: 2^{N} \backslash\{\emptyset\} \rightarrow N \mid \alpha(T) \in T\right.$ for all $\left.T \in 2^{N} \backslash\{\emptyset\}\right\}$ being the set of all selectors on $N$, and $\operatorname{Conv}(\{A\})$ denoting the convex hull of $A \subset \mathbb{R}^{n}$.

For TU-games $v \in \mathcal{G}^{N}$, Vasil'ev $(1978,1981)$ defines the Harsanyi set $H(v)$ as the set of payoff vectors obtained by distributing for any coalition the dividend of that coalition in any possible way among its players, i.e., $H(v)$ is the weighted sum of sets $P^{T} \subset R_{+}^{n}$ given by

$$
H(v)=\sum_{T \in 2^{N} \backslash\{\emptyset\}} \Delta_{v}(T) P^{T},
$$

with, for $T \in 2^{N} \backslash \emptyset, P^{T}$ the set of sharing vectors $p^{T} \in \mathbb{R}_{+}^{n}$ defined as

$$
P^{T}=\left\{\begin{array}{l|l}
p^{T} \in \mathbb{R}_{+}^{n} & \begin{array}{l}
\text { (i) } p_{i}^{T}=0 \text { for all } i \in N \backslash T, \\
\text { (ii) } p_{i}^{T} \geq 0 \text { for all } i \in T, \text { and } \\
\text { (iii) } \sum_{i \in T} p_{i}^{T}=1
\end{array}
\end{array}\right\} .
$$

\footnotetext{
${ }^{1}$ It is well-known that $C(N, v)$ is nonempty if and only if $v$ is balanced, see e.g. Bondareva (1962) or Shapley (1967), and is equal to the convex hull of all the marginal vectors of the game if and only if the game is convex, see Shapley (1971) and Ichiishi (1981).
} 
The set $\Delta_{v}(T) P^{T}$ gives any possible distribution of $\Delta_{v}(T)$ amongst the players in $T$ and $H(v)$ is obtained by summing up all these payoff sets over all nonempty sets $T$. An element of $H(v)$ is called a Harsanyi payoff vector of game $v$.

The Harsanyi set and Selectope are equivalent, i.e., $H(v)=S(v)$ for every $v \in \mathcal{G}^{N}$. In Vasil'ev (1981) (see also Derks et. al. (2000) and Vasil'ev and Van der Laan (2002)) it is shown that (i) for every TU-game $v \in \mathcal{G}^{N}$ it holds that $\operatorname{Core}(v) \subseteq H(v)$ with equality if and only if $v$ is almost positive, and (ii) $H(v)=\operatorname{Core}\left(v_{H}\right)$, with $v_{H}$ the convex game defined by $v_{H}(S)=v(S)+\sum_{\{T|| T \cap S|\geq 1, T \cap(N \backslash S)| \geq 1\}} \max \left[0,-\Delta_{v}(T)\right]$. The second assertion implies that the Harsanyi set has a convex game Core-type structure and thus is not empty. The latter fact also follows straightforwardly from the Harsanyi set always containing the Shapley value (Shapley (1953)) being the single-valued solution Sh: $\mathcal{G}^{N} \rightarrow \mathbb{R}^{N}$ given by

$$
S h_{i}(v)=\sum_{\{S \subseteq N \mid i \in S\}} \frac{\Delta_{v}(S)}{|S|} \text { for every } i \in N .
$$

So, for every game $v$ the Shapley value yields the Harsanyi payoff vector that is obtained by taking the sharing vectors $p^{T}$ given by $p_{i}^{T}=\frac{1}{|T|}$ for all $i \in T$ and every nonempty $T \subseteq N$.

\section{$2.2 \quad$ Directed graphs}

In this paper we assume the players to be part of a hierarchical structure that is represented by a directed graph. A directed graph or digraph is a pair $(N, D)$ where $N=\{1, \ldots, n\}$ is a finite set of nodes (representing the players) and $D \subseteq N \times N$ is a binary relation on $N$. We assume $D$ to be irreflexive, i.e. $(i, i) \notin D$ for all $i \in N$. The collection of all (irrefelxive) binary relations on $N$ is denoted by $\mathcal{D}^{N}$. Since we assume the finite set $N$ to be fixed, we will refer to a binary relation simply as a digraph on $N$. For a subset $A \subseteq D$ and $i \in N$, the nodes in $S_{A}(i):=\{j \in N \mid(i, j) \in A\}$ are called the successors of $i$ in $A$, and the nodes in $R_{A}(i):=\{j \in N \mid(j, i) \in A\}$ are called the predecessors of $i$ in $A$.

For $i, j \in N$, a path between $i$ and $j$ in $D$ is a sequence of nodes $\left(i_{1}, \ldots, i_{m}\right)$ such that $i_{1}=i, i_{m}=j$, and $\left\{\left(i_{k}, i_{k+1}\right),\left(i_{k+1}, i_{k}\right)\right\} \cap D \neq \emptyset$ for $k=1, \ldots, m-1$. A set of nodes $T \subseteq N$ is connected in $D \in \mathcal{D}^{N}$ if there is a path between any two nodes in $T$ that only uses arcs between nodes in $T$, i.e., if for every $i, j \in T$ there is a path $\left(i_{1}, \ldots, i_{m}\right)$ between $i$ and $j$ such that $\left\{i_{1}, \ldots, i_{m}\right\} \subseteq T$. A path $\left(i_{1}, \ldots, i_{m}\right)$ between $i$ and $j$ in $D$ is a directed path if $\left(i_{k}, i_{k+1}\right) \in D$ for $k=1, \ldots, m-1$. A directed path $\left(i_{1}, \ldots, i_{m}\right), m \geq 3$, in $D$ is a cycle in $D$ if $i_{1}=i_{m}$. We call digraph $D$ acyclic if it does not contain any cycle. Finally, a nonempty set $S \in 2^{N}$ is called comprehensive from above in $D$ if $[j \in S$ and $(i, j) \in D]$ implies that $i \in S$. A nonempty set $S \in 2^{N}$ is called complete in $D$ when $S$ is connected and comprehensive from above in $D$. We define $\mathcal{C}_{D}^{T}$ to be the set of complete subsets of $T$, $T \subseteq N \backslash \emptyset$ in $(T, D(T))$ where $D(T)=\{(i, j) \in D \mid\{i, j\} \subseteq T\}$. We define $D^{-1}$ to be the 
transpose of $D$, i.e., $D^{-1}=\{(i, j) \in N \times N \mid(j, i) \in D\}$. Then $\mathcal{C}_{D^{-1}}^{T}$ is the collection of complete subsets of $T$ in $D^{-1}$, i.e., a nonempty set $S \subseteq T$ is in $\mathcal{C}_{D^{-1}}^{T}$ if (i) $S$ is connected in $D$ and (ii) $S$ is comprehensive from below in $D$ meaning that $[j \in S$ and $(j, i) \in D]$ implies that $i \in S$.

\section{The Restricted Harsanyi set solution}

In this section we propose a modification of the Harsanyi set for situations in which players in a TU-game are part of a hierarchical structure represented by a digraph. A pair $(v, D) \in$ $\mathcal{G}^{N} \times \mathcal{D}^{N}$ is called a game with ordered players on $N$. A (set-valued) solution $\mathcal{F}$ on $\mathcal{G}^{N} \times \mathcal{D}^{N}$ assigns a set $\mathcal{F}(v, D) \subset \mathbb{R}^{n}$ of payoff vectors to every game with ordered players $(v, D)$.

Applying the idea of the Harsanyi set to games with ordered players we now take into account the position of the players within the graph when distributing the dividends of the coalitions by requiring that if $i, j \in T$ and $(i, j) \in D$, then the payoff that the dominating player $i$ receives from the distribution of the dividend $\Delta_{v}(T)$ is at least as high as the payoff that the dominated player $j$ receives from $\Delta_{v}(T)$. So, for $i, j \in T$ and $(i, j) \in D$ we require that $p_{i}^{T} \geq p_{j}^{T}$ when $\Delta_{v}(T)>0$, and that $p_{i}^{T} \leq p_{j}^{T}$ when $\Delta_{v}(T)<0 .{ }^{2}$ For a game $v$, define $K_{v}^{+}=\left\{T \mid \Delta_{v}(T)>0\right\}$ and $K_{v}^{-}=\left\{T \mid \Delta_{v}(T)<0\right\}$ as the sets of coalitions with positive, respectively, negative dividends in $v$. Further, let $P_{D}^{+T}$ and $P_{D}^{-T}$ be subsets of $P^{T}$ given by

$$
P_{D}^{+T}=\left\{p^{T} \in P^{T} \mid p_{i}^{T} \geq p_{j}^{T} \text { for all } i, j \in T \text { with }(i, j) \in D\right\},
$$

respectively

$$
P_{D}^{-T}=\left\{p^{T} \in P^{T} \mid p_{i}^{T} \leq p_{j}^{T} \text { for all } i, j \in T \text { with }(i, j) \in D\right\} .
$$

Definition 3.1 The Restricted Harsanyi set of game with ordered players $(v, D) \in \mathcal{G}^{N} \times$ $\mathcal{D}^{N}$ is the set

$$
\mathcal{H}(v, D)=\sum_{T \in K_{v}^{+}} \Delta_{v}(T) P_{D}^{+T}+\sum_{T \in K_{v}^{-}} \Delta_{v}(T) P_{D}^{-T} .
$$

The Restricted Harsanyi set solution is the solution that assigns the Restricted Harsanyi set to every game $(v, D) \in \mathcal{G}^{N} \times \mathcal{D}^{N}$. Since all sets $P_{D}^{+T}$ and $P_{D}^{-T}$ are polytopes, note that also $\mathcal{H}(v, D)$ is a polytope for any $(v, D)$. The definition of the Restricted Harsanyi Set implies that it always contains the Shapley value and is contained in the Harsanyi set of the game.

\footnotetext{
${ }^{2}$ The shares don't matter when $\Delta_{v}(T)=0$.
} 
Theorem 3.2 For every $(v, D) \in \mathcal{G}^{N} \times \mathcal{D}^{N}$ it holds that $\operatorname{Sh}(v) \in \mathcal{H}(v, D) \subseteq H(v)$.

Proof. Let $(v, D) \in \mathcal{G}^{N} \times \mathcal{D}^{N}$. Take $p_{i}^{T}=\frac{1}{|T|}$ if $i \in T$, and $p_{i}^{T}=0$ otherwise. Clearly, $\sum_{T \in 2^{N} \backslash\{\emptyset\}} \Delta_{v}(T) p^{T}=S h(v)$. Further, for any $T$ we have that $p^{T} \in P_{D}^{+T} \cap P_{D}^{-T}$ for every $D \in \mathcal{D}^{N}$. Hence $\operatorname{Sh}(v) \in \mathcal{H}(v, D)$. The inclusion $\mathcal{H}(v, D) \subseteq H(v)$ follows immediately from the fact that both $P_{D}^{+T} \subseteq P^{T}$ and $P_{D}^{-T} \subseteq P^{T}$.

Note that $\operatorname{Sh}(v) \in \mathcal{H}(v, D)$ implies that $\mathcal{H}(v, D) \neq \emptyset$ for all $(v, D) \in \mathcal{G}^{N} \times \mathcal{D}^{N}$. The Restricted Harsanyi set $\mathcal{H}(v, D)$ generalizes both the Shapley value and the Harsanyi set for TU-games, in the sense that the corresponding inclusions in Theorem 3.2 can be equalities.

Theorem 3.3 Let $D \in \mathcal{D}^{N}$. Then

(i) $\mathcal{H}(v, D)=\{S h(v)\}$ for every $v \in \mathcal{G}^{N}$ if and only if $D=N \times N$;

(ii) $\mathcal{H}(v, D)=H(v)$ for every $v \in \mathcal{G}^{N}$ if and only if $D=\emptyset$.

Proof. (i) If Take $D=N \times N$. Then $(i, j) \in D$ for all $i, j \in N$, and thus $P_{D}^{+T}=P_{D}^{-T}=$ $\left\{p^{T} \in P^{T} \mid p_{i}^{T}=p_{j}^{T}\right.$ for all $\left.i, j \in T\right\}=\left\{p^{T} \in P^{T} \mid p_{i}^{T}=\frac{1}{|T|}\right.$ for all $\left.i \in T\right\}$ for any $T \neq \emptyset$. So $\mathcal{H}(v, D)=\{S h(v)\}$ for every $v \in \mathcal{G}^{N}$. Only if Suppose that $D \neq N \times N$, i.e., there exist $i, j \in N, i \neq j$, with $(i, j) \notin D$. Take $v=u_{\{i, j\}}$ and let $e^{j} \in \mathbb{R}^{N}$ be given by $e_{j}^{j}=1$ and $e_{h}^{j}=0$ for all $h \in N \backslash\{j\}$. Then $e^{j} \in \mathcal{H}(v, D)$ although $e^{j} \neq S h(v)$.

(ii) If Take $D=\emptyset$. Then $P_{D}^{+T}=P_{D}^{-T}=P^{T}$ for any $T \neq \emptyset$, implying that $\mathcal{H}(v, D)=H(v)$. Only if Suppose that $D \neq \emptyset$, i.e., there exist $i, j \in N, i \neq j$, with $(i, j) \in D$. Again, take $v=u_{\{i, j\}}$ and consider $e^{j} \in \mathbb{R}^{N}$. Then $e^{j} \notin \mathcal{H}(v, D)$ although $e^{j} \in H(v)$.

To find the extreme points of the Restricted Harsanyi set, the following theorem from van den Brink et. al. (2004) about the extreme points of the set $P_{D}^{+T}$ is useful. For $S \subseteq N$, let $a^{S} \in \mathbb{R}^{n}$ be the vector with components $a_{i}^{S}=\frac{1}{|S|}$ when $i \in S$ and $a_{i}^{S}=0$, otherwise. The lemma says that $a^{S}$ is an extreme point of $P_{D}^{+T}$ if and only if $S$ is a complete subset of $T$. For the proof we refer to van den Brink et. al. (2004).

Theorem 3.4 For $T \subseteq N$, the set of extreme points of $P_{D}^{+T}$ is given by

$$
\operatorname{Ex}\left(P_{D}^{+T}\right)=\left\{a^{S} \in \mathbb{R}^{n} \mid S \in \mathcal{C}_{D}^{T}\right\} .
$$

For the extreme points of $P_{D}^{-T}$ the next corollary follows straightforwardly from Theorem 3.4 and is given without proof.

Corollary 3.5 The set of extreme points of $P_{D}^{-T}$ is given by

$$
\operatorname{Ex}\left(P_{D}^{-T}\right)=\left\{a^{S} \in \mathbb{R}^{n} \mid S \in \mathcal{C}_{D^{-1}}^{T}\right\} .
$$


We conclude this section with some examples in which we apply Theorem 3.4 to find the extreme points.

Example 3.6 (i) Consider the game with ordered players $(v, D)$ on $N=\{1,2,3\}$ given by $v=u_{\{1,2\}}+u_{\{1,3\}}$ and $D=\{(1,3),(2,3)\}$. First observe that $H(v)=P^{\{1,2\}}+P^{\{1,3\}}=$ $\operatorname{Conv}\left(\left\{(1,0,0)^{\top},(0,1,0)^{\top}\right\}\right)+\operatorname{Conv}\left(\left\{(1,0,0)^{\top},(0,0,1)^{\top}\right\}\right)=$ $\operatorname{Conv}\left(\left\{(2,0,0)^{\top},(1,1,0)^{\top},(1,0,1)^{\top},(0,1,1)^{\top}\right\}\right)$. The Restricted Harsanyi set is equal to $\mathcal{H}(v, D)=P_{D}^{+\{1,2\}}+P_{D}^{+\{1,3\}}=P^{\{1,2\}}+\left\{p^{\{1,3\}} \in P^{\{1,3\}} \mid p_{1}^{\{1,3\}} \geq p_{3}^{\{1,3\}}\right\}$. It follows that $\mathcal{H}(v, D)=\operatorname{Conv}\left(\left\{(1,0,0)^{\top},(0,1,0)^{\top}\right\}\right)+\operatorname{Conv}\left(\left\{(1,0,0)^{\top},\left(\frac{1}{2}, 0, \frac{1}{2}\right)^{\top}\right\}\right)=$ $\operatorname{Conv}\left(\left\{(2,0,0)^{\top},(1,1,0)^{\top},\left(1 \frac{1}{2}, 0, \frac{1}{2}\right)^{\top},\left(\frac{1}{2}, 1, \frac{1}{2}\right)^{\top}\right\}\right)$, see Figure 1 .

(ii) Consider the game with ordered players $(v, D)$ on $N=\{1,2,3\}$ given by $v=2 u_{\{1,2\}}-$ $u_{\{2,3\}}$ and $D=\{(1,2),(2,3)\}$. In this case we have that $H(v)=2 P^{\{1,2\}}-P^{\{2,3\}}=$ $\operatorname{Conv}\left(\left\{(2,0,0)^{\top},(0,2,0)^{\top}\right\}\right)+\operatorname{Conv}\left(\left\{(0,-1,0)^{\top},(0,0,-1)^{\top}\right\}\right)=$

$\operatorname{Conv}\left(\left\{(2,-1,0)^{\top},(0,1,0)^{\top},(2,0,-1)^{\top},(0,2,-1)^{\top}\right\}\right)$. The Restricted Harsanyi set is equal to $\mathcal{H}(v, D)=2 P_{D}^{+\{1,2\}}-P_{D}^{-\{2,3\}}=2\left\{p^{\{1,2\}} \in P^{\{1,2\}} \mid p_{1}^{\{1,2\}} \geq p_{2}^{\{1,2\}}\right\}-\left\{p^{\{2,3\}} \in P^{\{2,3\}} \mid p_{2}^{\{2,3\}} \leq\right.$ $\left.p_{3}^{\{2,3\}}\right\}=\operatorname{Conv}\left(\left\{(2,0,0)^{\top},(1,1,0)^{\top}\right\}+\operatorname{Conv}\left\{\left(0,-\frac{1}{2},-\frac{1}{2}\right)^{\top},(0,0,-1)^{\top}\right\}\right)=$ $\operatorname{Conv}\left(\left\{\left(2,-\frac{1}{2},-\frac{1}{2}\right)^{\top},\left(1, \frac{1}{2},-\frac{1}{2}\right)^{\top},(2,0,-1)^{\top},(1,1,-1)^{\top}\right\}\right)$.

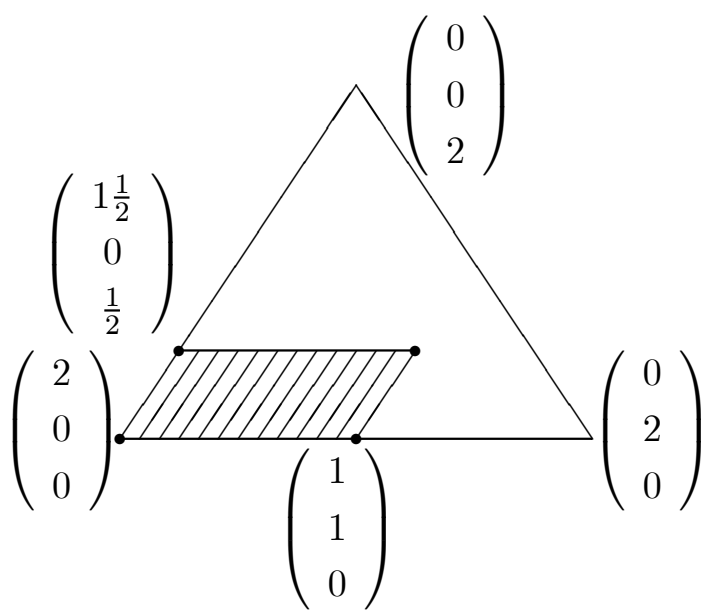

Figure 1: $\mathcal{H}(v, D)$ of Example 3.6.(i).

\section{Axiomatic characterization of the Restricted Harsanyi set solution}

In this section we characterize the Restricted Harsanyi set solution as the unique nonempty solution on $\mathcal{G}^{N} \times \mathcal{D}^{N}$ satisfying a collection of seven logically independent axioms. Let $\mathcal{F}$ 
be a solution on $\mathcal{G}^{N} \times \mathcal{D}^{N}$ assigning a set $\mathcal{F}(v, D)$ of payoff vectors to any game $(v, D)$. The first five axioms are generalizations of standard axioms in cooperative game theory, and are discussed for TU-games in Vasil'ev and van der Laan (2002).

Axiom (Convexity) The set $\mathcal{F}(v, D) \subset \mathbb{R}^{n}$ is convex.

Axiom (Efficiency) Any payoff vector in the solution is efficient, i.e. $\sum_{i \in N} x_{i}=v(N)$ for all $x \in \mathcal{F}(v, D)$.

Recall that a player $i \in N$ is a null player in $v \in \mathcal{G}^{N}$ if $v(S)=v(S \backslash\{i\})$ for all $S \subseteq N$.

Axiom (Null player property) Any payoff vector $x \in \mathcal{F}(v, D)$ satisfies $x_{i}=0$, whenever $i$ is a null player in $v$.

Two games $v, w \in \mathcal{G}^{N}$ are called disjoint if $\Delta_{v}(T) \Delta_{w}(T)=0$ for all $T \in 2^{N} \backslash\{\emptyset\}$, i.e. any coalition has a nonzero dividend in at most one of these two games.

Axiom (Disjoint additivity) If $v$ and $w$ are disjoint games, then $\mathcal{F}(v+w, D)=$ $\mathcal{F}(v, D)+\mathcal{F}(w, D)$.

Let $V^{+} \subset \mathcal{G}^{N}$ be the collection of totally positive games (all dividends are nonnegative) and $V^{-} \subset \mathcal{G}^{N}$ the collection of totally negative games (all dividends are nonpositive). The next axiom states that in games with nonnegative (respectively nonpositive) dividends all payoffs are nonnegative (respectively nonpositive).

Axiom (Sign preservation) The set $\mathcal{F}(v, D) \subseteq \mathbb{R}_{+}^{n}$ if $v \in V^{+}$, and $\mathcal{F}(v, D) \subseteq \mathbb{R}_{-}^{n}$ if $v \in V^{-}$.

Next we introduce two new properties. The first one reflects the hierarchical dominance. It states that players always earn at least as much as players that are inferior to them. We call player $j$ inferior to player $i$ in $(v, D) \in \mathcal{G}^{N} \times \mathcal{D}^{N}$ if $(i, j) \in D$ and $\Delta_{v}(T)=0$ if $T \in \Omega_{\{i, j\}}=\{T \subset N||\{i, j\} \cap T \mid=1\}$ being the set of all subsets $T$ of $N$ that either contains $i$ or $j$ (but not both).

Axiom (Inferior player property) Any payoff vector $x \in \mathcal{F}(v, D)$ satisfies $x_{i} \geq x_{j}$ whenever $j$ is inferior to $i$. 
Observe that it may happen that both $(i, j) \in D$ and $(j, i) \in D$ and that $\Delta_{v}(T)=0$ for all $T \in \Omega_{\{i, j\}}$. In this case $i$ is inferior to $j$ and $j$ is inferior to $i$, so that the inferior player property says that $x_{i}=x_{j}$ for any $x \in \mathcal{F}(v, D)$.

The last property is a consistency property. For any pair of nonempty sets $T \subseteq N$ and $R \subseteq T$ we define the game $v_{R, T}$ by

$$
v_{R, T}(S)= \begin{cases}v(S) & \text { if } T \subseteq S, \\ v(S)+\frac{|R \cap S|}{|R|} \Delta_{v}(T) & , \text { otherwise. }\end{cases}
$$

The next proposition follows by straightforward calculation of the dividends and is given without proof.

Proposition 4.1 For $T \subseteq N$ and $R \subseteq T$ the dividends of game $v_{R, T}$ are given by

$$
\Delta_{v_{R}, T}(S)= \begin{cases}\Delta_{v}(S)+\frac{1}{|R|} \Delta_{v}(T) & \text { if } S \subseteq R,|S|=1 \\ 0 & \text { if } S=T \\ \Delta_{v}(S) & \text { otherwise. }\end{cases}
$$

So, in terms of the dividends the game $v_{R, T}$ is obtained from the original game by distributing the dividend of coalition $T$ equally amongst the players in $R$. Recall that a selector chooses for any nonempty coalition one of the players in that coalition as some representative to whom to assign the dividend of that coalition. In the game $v_{R, T}$ the coalition $T$ becomes a 'null coalition' in the sense that its dividend is zero, while the players in $R$ are chosen to represent $T$ meaning that they all individually get an equal share in the dividend of $T$.

Recall that $\mathcal{C}_{D}^{T}$ is the collection of subsets of $T$ that are connected and comprehensive from above in $D$, while $\mathcal{C}_{D^{-1}}^{T}$ is the collection of subsets of $T$ that are connected and comprehensive from below in $D$.

Axiom (Coalitional consistency property) Consider $T \subseteq N, T \neq \emptyset$. For all $R \in \mathcal{C}_{D}^{T}$, $\mathcal{F}\left(v_{R, T}, D\right) \subseteq \mathcal{F}(v, D)$ if $\Delta_{v}(T)>0$; for all $R \in \mathcal{C}_{D^{-1}}^{T}, \mathcal{F}\left(v_{R, T}, D\right) \subseteq \mathcal{F}(v, D)$ if $\Delta_{v}(T)<0$.

This property states that by allocating the dividend of a coalition equally among some of the players in that coalition, we do not obtain new payoff vectors in the solution. Going from a game $v$ with ordered players to the game $v_{R, T}$ with the same ordering of the players, we don not yet assign payoffs to the players, but we allocate one of the coalitional dividends to singleton dividends. Considering this as a partial solution, then the consistency requirement is that the payoff vectors assigned to the new game should also be payoff vectors in the original game.

To prove that $\mathcal{H}(v, D)$ is characterized by the seven axioms stated above, we first state three lemmas. In the sequel $s T$ denotes $+T$ when $s=1$, and $-T$ when $s=-1$. 
Lemma 4.2 Let $\mathcal{F}$ be a solution on $\mathcal{G}^{N} \times \mathcal{D}^{N}$ satisfying efficiency, the null player property, sign preservation and the inferior player property. Then for any $D \in \mathcal{D}^{N}$ and nonempty $T \subseteq N$, we have that $\mathcal{F}\left(u_{T}, D\right) \subseteq P_{D}^{+T}$ and $\mathcal{F}\left(-u_{T}, D\right) \subseteq-P_{D}^{-T}$.

Proof. Let $x \in \mathcal{F}\left(s u_{T}, D\right), s \in\{-1,+1\}$. Then, from efficiency it follows that $\sum_{i \in N} x_{i}=$ $s$ and by sign preservation we have that $x_{i} \geq 0, i \in N$, when $s=+1$, respectively $x_{i} \leq 0$, $i \in N$, when $s=-1$. From the null player property we have that $x_{i}=0$ if $i \in N \backslash T$. Finally, from the inferior player property we obtain that for any two players $i, j \in T$ with $(i, j) \in D$ it holds that $x_{i} \geq x_{j}$. Hence $\mathcal{F}\left(s u_{T}, D\right) \subseteq s P_{D}^{s T}$.

In the proof of the next lemma we apply Theorem 3.4 and its Corollary 3.5 on the extreme points of $P_{D}^{+T}$, respectively, $P_{D}^{-T}$.

Lemma 4.3 Let $\mathcal{F}$ be a nonempty solution on $\mathcal{G}^{N} \times \mathcal{D}^{N}$ satisfying convexity, efficiency, the null player property, disjoint additivity and coalitional consistency. Then for any $D \in \mathcal{D}^{N}$ and nonempty $T \subseteq N$, we have that $P_{D}^{+T} \subseteq \mathcal{F}\left(u_{T}, D\right)$ and $-P_{D}^{-T} \subseteq \mathcal{F}\left(-u_{T}, D\right)$.

Proof. It follows from Proposition 4.1 that for $R \subseteq T$ the game $s w_{R, T}=\left(s u_{T}\right)_{R, T}$ satisfies

$$
s w_{R, T}=\sum_{i \in R} \frac{1}{|R|} s u_{\{i\}} .
$$

From the non-emptiness, efficiency and the null player property it follows that for any $D, \mathcal{F}\left(\frac{1}{|R|} s u_{\{i\}}, D\right)=\frac{1}{R} s a^{\{i\}}, i \in N$. Then disjoint additivity implies that $\mathcal{F}\left(s w_{R, T}, D\right)=$ $\sum_{i \in R} \mathcal{F}\left(\frac{1}{|R|} s u_{\{i\}}, D\right)=\sum_{i \in R} \frac{1}{R} s a^{\{i\}}=s a^{R}$. Hence coalitional consistency implies that $a^{R} \in \mathcal{F}\left(u_{T}, D\right)$ for any $R \in \mathcal{C}_{D}^{T}$, and $-a^{R} \in \mathcal{F}\left(-u_{T}, D\right)$ for any $R \in \mathcal{C}_{D^{-1}}^{T}$. Finally, with Theorem 3.4, respectively Corollary 3.5, convexity implies that $P_{D}^{+T}=\operatorname{Conv}\left(\left\{a^{R}, R \in\right.\right.$ $\left.\left.\mathcal{C}_{D}^{T}\right\}\right) \subseteq \mathcal{F}\left(u_{T}, D\right)$ and $-P_{D}^{-T}=\operatorname{Conv}\left(\left\{-a^{R}, R \in \mathcal{C}_{D^{-1}}^{T}\right\}\right) \subseteq \mathcal{F}\left(-u_{T}, D\right)$

Lemma 4.4 Let $\mathcal{F}$ be a nonempty solution on $\mathcal{G}^{N} \times \mathcal{D}^{N}$ satisfying convexity, efficiency, the null player property, disjoint additivity, sign preservation, the inferior player property and coalitional consistency. Then for any $D \in \mathcal{D}^{N}$, nonempty $T \subseteq N$ and $c>0$, we have that $\mathcal{F}\left(c u_{T}, D\right)=c P_{D}^{+T}$ and $\mathcal{F}\left(-c u_{T}, D\right)=c\left(-P_{D}^{-T}\right)$.

Proof. For $c=1$, the statement follows immediately from Lemmas 4.2 and 4.3. For positive $c \neq 1$ it follows from the fact that the set of extreme points of $\operatorname{cs} P_{D}^{s T}$ is given by $\operatorname{Ex}\left(c s P_{D}^{s T}\right)=c s \operatorname{Ex}\left(P_{D}^{s T}\right), s \in\{-1,+1\}$. The result then follows by repeating the reasoning given in the Lemmas 4.2 and 4.3 for the games $c s u_{T}$.

We now come to the main theorem. 
Theorem 4.5 A nonempty solution $\mathcal{F}$ on $\mathcal{G}^{N} \times \mathcal{D}^{N}$ satisfies convexity, efficiency, the null player property, disjoint additivity, sign preservation, the inferior player property and coalitional consistency, if and only if $\mathcal{F}$ is the Restricted Harsanyi set solution.

Proof. It is straightforward to verify that the (nonempty) Restricted Harsanyi set solution satisfies these properties. To show uniqueness, suppose that a nonempty solution $\mathcal{F}$ on $\mathcal{G}^{N} \times \mathcal{D}^{N}$ satisfies the seven axioms. From disjoint additivity we have that

$$
\begin{aligned}
\mathcal{F}(v, D) & =\sum_{T \in 2^{N} \backslash \emptyset} \mathcal{F}\left(\Delta_{v}(T) u_{T}, D\right) \\
& =\sum_{T \in K_{v}^{+}} \mathcal{F}\left(\Delta_{v}(T) u_{T}, D\right)+\sum_{T \in K_{v}^{-}} \mathcal{F}\left(\Delta_{v}(T) u_{T}, D\right) .
\end{aligned}
$$

With Lemma 4.4 it then follows that

$$
\mathcal{F}(v, D)=\sum_{T \in K_{v}^{+}} \Delta_{v}(T) P_{D}^{+T}+\sum_{T \in K_{v}^{-}} \Delta_{v}(T) P_{D}^{-T}=\mathcal{H}(v, D) .
$$

We show logical independence of the seven axioms in Theorem 4.5 by the following alternative solutions on $\mathcal{G}^{N} \times \mathcal{D}^{N}$.

1. Solution $\mathcal{F}_{C}$ given by $\mathcal{F}_{C}(v, D)=\sum_{T \in K_{v}^{+}} \Delta_{v}(T) \operatorname{Ex}\left(P_{D}^{+T}\right)+\sum_{T \in K_{v}^{-}} \Delta_{v}(T) \operatorname{Ex}\left(P_{D}^{-T}\right)$ satisfies the axioms of Theorem 4.5 except convexity.

2. Solution $\mathcal{F}_{E}$ given by $\mathcal{F}_{E}(v, D)=\mu \mathcal{H}(v, D)$ for some positive $\mu \neq 1$, satisfies the axioms of Theorem 4.5 except efficiency.

3. Define $\bar{v} \in \mathbb{R}^{n}$ by $\bar{v}_{i}=\frac{1}{|N|} v(N)$. Solution $\mathcal{F}_{N P}$ given by $\mathcal{F}_{N P}(v, D)=\mu\{\bar{v}\}+(1-$ $\mu) \mathcal{H}(v, D)$, for some $\mu, 0<\mu<1$, satisfies the axioms of Theorem 4.5 except the null player property.

4. Let $\underline{0}$ denote the null-vector in $\mathbb{R}^{n}$. Solution $\mathcal{F}_{D A}$ given by $\mathcal{F}_{D A}(v, D)=\{\underline{0}\}$ when $v(N)=0$ and $\mathcal{F}_{D A}(v, D)=\mathcal{H}(v, D)$ otherwise, satisfies the axioms of Theorem 4.5 except disjoint additivity.

5. For some $\epsilon>0$, define $P^{T}(\epsilon)=\left\{p^{T} \in \mathbb{R}^{n} \mid p_{i}^{T}=0\right.$, if $i \notin T, p_{i}^{T} \geq-\epsilon$ if $i \in T$, and $\left.\sum_{i \in T} p_{i}^{T}=1, T \in 2^{N} \backslash\{\emptyset\}\right\}$. Let $P_{D}^{+T}(\epsilon)$ and $P_{D}^{-T}(\epsilon)$ be as defined before, but with $P^{T}(\epsilon)$ instead of $P^{T}$, and define the solution $\mathcal{H}^{\epsilon}$ by $\mathcal{H}^{\epsilon}(v, D)=$ $\sum_{T \in K_{v}^{+}} \Delta_{v}(T) P_{D}^{+T}(\epsilon)+\sum_{T \in K_{v}^{-}} \Delta_{v}(T) P_{D}^{-T}(\epsilon)$. Solution $\mathcal{F}_{S P}$ given by $\mathcal{F}_{S P}(v, D)=$ $\mathcal{H}^{\epsilon}(v, D)$ satisfies the axioms of Theorem 4.5 except sign preservation.

6. Solution $\mathcal{F}_{I P}$ given by $\mathcal{F}_{I P}(v, D)=\mathcal{H}(v, D)$ satisfies the axioms of Theorem 4.5 except the inferior player property. 
7. Solution $\mathcal{F}_{R G}$ given by $\mathcal{F}_{R G}(v, D)=\{S h(v)\}$ satisfies the axioms of Theorem 4.5 except coalitional consistency.

The results stated above imply several corollaries for games with ordered players and for (unrestricted) TU-games. First, from Lemma 4.2, Lemma 4.4 and Theorem 4.5 it follows that for any solution $\mathcal{F}$ satisfying efficiency, the null player property, sign preservation and the inferior player property, it holds that $\mathcal{F}\left(s c u_{T}, D\right) \subseteq c s P_{D}^{s T}=\mathcal{H}\left(s c u_{T}, D\right), s \in$ $\{-1,+1\}$. From this it follows that $\mathcal{F}(v, D) \subseteq \mathcal{H}(v, D)$ for any solution $\mathcal{F}$ that satisfies these four axioms and disjoint additivity. So, the Restricted Harsanyi set solution is the maximal (with respect to set inclusion) solution that satisfies efficiency, the null player property, sign preservation, the inferior player property and disjoint additivity.

Second, let $\mathcal{F}^{\mathrm{Conv}}$ and $\mathcal{F}^{\mathrm{Cl}}$ be the solutions that assign to every game with ordered players $(v, D)$ the sets $\operatorname{Conv}(\mathcal{F}(v, D))$, respectively $\operatorname{Cl}(\mathcal{F}(v, D))$, where $\operatorname{Cl}(A)$ denotes the closure of set $A$. Then it is easy to verify that when $\mathcal{F}$ satisfies efficiency, the null player property, sign preservation, the inferior player property and disjoint additivity, then also $\mathcal{F}^{\mathrm{Conv}}$ and $\mathcal{F}^{\mathrm{Cl}}$ satisfy these five axioms. ${ }^{3}$ Hence, when $\mathcal{F}$ satisfies efficiency, the null player property, sign preservation, the inferior player property and disjoint additivity, then also $\operatorname{Conv}(\mathcal{F}(v, D)) \subseteq \mathcal{H}(v, D)$ and $\operatorname{Cl}(\mathcal{F}(v, D)) \subseteq \mathcal{H}(v, D)$.

These results for games with ordered players are summarized in the next corollary.

Corollary 4.6 On the class $\mathcal{G}^{N} \times \mathcal{D}^{N}$ we have that:

(i) $\mathcal{F}(v, D) \subseteq \mathcal{H}(v, D)$ for any $(v, D)$ when $\mathcal{F}$ satisfies efficiency, the null player property, sign preservation, the inferior player property and disjoint additivity;

(ii) When the solution $\mathcal{F}$ satisfies the efficiency, the null player property, sign preservation, the inferior player property and disjoint additivity, then also the solutions $\mathcal{F}^{C o n v}$ and $\mathcal{F}^{C l}$ satisfy these properties.

When $D=\emptyset$ the inferior player property gives no restriction. Defining $F(v)=$ $\mathcal{F}(v, \emptyset)$ as a solution for TU-games (i.e. a solution on $\mathcal{G}^{N}$ ), then $F(v) \subseteq H(v)$ when $F$ satisfies efficiency, the null player property, sign preservation and disjoint additivity. Moreover, Theorem 4.5 gives a characterization of the Harsanyi set for TU-games as the unique nonempty solution on $\mathcal{G}^{N}$ that satisfies convexity, efficiency, the null player property, sign preservation, coalitional consistency and disjoint additivity ${ }^{4}$. Further, note that in this case $R \subseteq T$ is complete in $D$, respectively in $D^{-1}$, if and only if $R$ is a singleton coalition.

\footnotetext{
${ }^{3}$ Disjoint additivity follows from the well-known fact that for any two sets $A, B \subset \mathbb{R}^{n}$, it holds that $\operatorname{Conv}(A+B)=\operatorname{Conv}(A)+\operatorname{Conv}(B)$, respectively, when at least one of the sets is bounded, $\operatorname{Cl}(A+B)=$ $\mathrm{Cl}(A)+\mathrm{Cl}(B)$.

${ }^{4}$ Note that on the class of games with ordered players all these axioms are defined for a fixed $D \in \mathcal{D}^{N}$. Here we refer to corresponding axioms on the class of TU-games which are obtained by taking $D=\emptyset$.
} 
So, coalitional consistency reduces to $\mathcal{F}\left(v_{R, T}, D\right) \subseteq \mathcal{F}(v, D)$ for all $R=\{i\} \subseteq T$, i.e., only the games $v_{R, T}$ in which the dividend of coalition $T$ is fully given to some player in $T$ are considered. This axiom replaces the individually consistency axiom in the characterization of the Harsanyi set given in Vasil'ev and van der Laan (2002), while the other five axioms are the same.

Finally, when $D=N \times N$, the inferior player property implies unanimity symmetry (i.e. in a unanimity game all players in the unanimity coalition get the same). Moreover, in this case $R$ is comprehensive from above, respectively below, in $T$ given $D$ if and only if $R=T$. Hence, coalitional consistency reduces to $\mathcal{F}\left(v_{R, T}, D\right) \subseteq \mathcal{F}(v, D)$ for $R=T$, i.e., only the game $v_{R, T}$ in which the dividend of coalition $T$ is equally divided amongst the players in $T$ are considered. For unanimity games this also reduces to unanimity symmetry. Since $\mathcal{H}(v, D)=\{S h(v)\}$ when $D=N \times N$, we obtain a characterization of the Shapley value as the unique single-valued solution satisfying efficiency, the null player property, disjoint additivity and unanimity symmetry. Note that disjoint additivity and unanimity symmetry are weaker than the corresponding axioms used in the famous characterization of the Shapley value in Shapley (1953). Also note that in this case sign preservation (although satisfied by the Shapley value) is superfluous in the characterization ${ }^{5}$.

These results for TU-games are summarized in the next corollary.

Corollary 4.7 On the class $\mathcal{G}^{N}$ of TU-games we have that:

(i) if solution $F$ on $\mathcal{G}^{N}$ satisfies efficiency, the null player property, sign preservation and disjoint additivity, then $F(v) \subseteq H(v)$ for all $v \in \mathcal{G}^{N}$;

(ii) the Harsanyi set solution $H$ is the unique nonempty solution on $\mathcal{G}^{N}$ that satisfies convexity, efficiency, the null player property, sign preservation, coalitional consistency for all $R=\{i\} \subseteq T, T \subseteq N$, and disjoint additivity;

(iii) the Shapley value is the unique single-valued solution on $\mathcal{G}^{N}$ that satisfies efficiency, the null player property, unanimity symmetry and disjoint additivity.

\section{Other solutions for games with ordered players}

The inferior player property reflects a very strong dominance of player $i$ above player $j$ when $(i, j) \in D$. In fact, it gives the dominating player not only the power to extract a higher share than the dominated player in the dividend of a coalition when that dividend is positive, but also the power to give the dominated player a higher share than the dominating player when the dividend is negative. To give an extreme example, let $D$ be a graph in which $(1, j) \in D$ and $(j, 1) \notin D$ for any $j \neq 1$. Then $x$ with $x_{1}=\sum_{\left\{T \mid 1 \in T, \Delta_{v}(T)>0\right\}} \Delta_{v}(T)$

\footnotetext{
${ }^{5}$ Convexity is not listed here, because this is redunded in case of single-valuedness.
} 
is in the Restricted Harsanyi set, giving player 1 all positive dividends of the coalitions containing player 1 , but nothing of the negative dividends.

Depending on the particular organizational (hierarchical) structure reflected by the digraph $D$, an alternative dominance relation would be in which the share of the dominating player in the dividend of a coalition is always at least as high as the share of a dominated player. For instance, this could reflect situations in which a dominating player takes higher risks than a dominated player and therefore get a higher share in positive dividends, but also in negative dividends.

This is reflected by the following notion and corresponding axiom. We call player $j$ weak inferior to player $i$ in $(v, D) \in \mathcal{G}^{N} \times \mathcal{D}^{N}$ if (i) $\Delta_{v}(T)=0$ if $T \in \Omega_{\{i, j\}}$ and (ii) either $(i, j) \in D$ and $\Delta_{v}(T) \geq 0$ for all $T$ containing $i$ and $j$, or $(j, i) \in D$ and $\Delta_{v}(T) \leq 0$ for all $T$ containing $i$ and $j$.

Axiom (Weak inferior player property) Any payoff vector $x \in \mathcal{F}(v, D)$ satisfies $x_{i} \geq x_{j}$ whenever $j$ is weak inferior to $i$.

The axiom says that the payoff to player $i$ is at least as high as the payoff to player $j$ if all dividends of the coalitions containing either $i$ or $j$ are zero and either $i$ dominates $j$ and all dividends of the coalitions containing $i$ and $j$ are nonnegative, or if $i$ is dominated by $j$ and all dividends of the coalitions containing $i$ and $j$ are nonpositive. Clearly, the axiom is satisfied when in any coalition containing $i$ and $j$, the share of player $i$ in the dividend is at least as high as the share of player $j$, irrespective whether the dividend is positive or negative. This holds when for any $T$ the sharing vector is in the set $P_{D}^{+T}$, irrespective of the sign of the dividend. This gives as alternative solution the Weak Inferior Player (WIP) Restricted Harsanyi set solution.

Definition 5.1 The WIP Restricted Harsanyi set solution is the solution $\mathcal{H}^{W}$ on $\mathcal{G}^{N} \times \mathcal{D}^{N}$ defined by

$$
\mathcal{H}^{W}(v, D)=\sum_{T \in 2^{N} \backslash \emptyset} \Delta_{v}(T) P_{D}^{+T},(v, D) \in \mathcal{G}^{N} \times \mathcal{D}^{N}
$$

Similarly as in the previous section the solution can be characterized by modifying coalitional consistency to sign-independent coalitional consistency.

Axiom (Sign-independent coalitional consistency) For all $R \in \mathcal{C}_{D}^{T}, \mathcal{F}\left(v_{R, T}, D\right) \subseteq$ $\mathcal{F}(v, D)$.

The next theorem characterizes the WIP Restricted Harsanyi set solution by similar axioms as those in Theorem 4.5. 
Theorem 5.2 A nonempty solution $\mathcal{F}$ on $\mathcal{G}^{N} \times \mathcal{D}^{N}$ satisfies convexity, efficiency, the null player property, sign preservation, the weak inferior player property, sign-independent coalitional consistency and disjoint additivity, if and only if $\mathcal{F}$ is the WIP Restricted Harsanyi set solution.

Since the proof goes along the same lines as the proof of Theorem 4.5, we leave it to the reader. In the next example we compare the two solutions.

Example 5.3 Consider game (ii) of Example 3.6, i.e., $N=\{1,2,3\}, v=2 u_{\{1,2\}}-u_{\{2,3\}}$ and $D=\{(1,2),(2,3)\}$. Then $\mathcal{H}^{W}(v)=2 P_{D}^{+\{1,2\}}-P_{D}^{+\{2,3\}}=$ $2\left\{p^{\{1,2\}} \in P^{\{1,2\}} \mid p_{1}^{\{1,2\}} \geq p_{2}^{\{1,2\}}\right\}-\left\{p^{\{2,3\}} \in P^{\{2,3\}} \mid p_{2}^{\{2,3\}} \geq p_{3}^{\{2,3\}}\right\}$. It follows that $\mathcal{H}^{W}(v, D)=\operatorname{Conv}\left(\left\{(2,0,0)^{\top},(1,1,0)^{\top}\right\}\right)+\operatorname{Conv}\left(\left\{\left(0,-\frac{1}{2},-\frac{1}{2}\right)^{\top},(0,-1,0)^{\top}\right\}\right)=$ $\operatorname{Conv}\left(\left\{\left(2,-\frac{1}{2},-\frac{1}{2}\right)^{\top},\left(1, \frac{1}{2},-\frac{1}{2}\right)^{\top},(2,-1,0)^{\top},(1,0,0)^{\top}\right\}\right)$.

Next we consider shortly how to apply the concept of selector to games with ordered players. Recall that a selector chooses for every coalition a particular player in the coalition to whom to assign the dividend of that coalition. In accordance with reasoning used in defining the Restricted Harsanyi set solution, when the players are ordered according to a digraph $D \in \mathcal{D}^{N}$ we put as restriction on a selector that the dividend of a coalition should always be assigned to a player that has no predecessors in the coalition when the dividend is positive, and to a player that has no successors in the coalition when the dividend is negative. So, we consider only selectors in the sets

$$
\mathcal{A}_{D}^{+N}=\left\{\alpha \in \mathcal{A}^{N} \mid \text { for every } T \in 2^{N} \backslash\{\emptyset\}:[\{i, j\} \subseteq T \text { and }(i, j) \in D] \Rightarrow \alpha(T) \neq j\right\},
$$

respectively

$$
\mathcal{A}_{D}^{-N}=\left\{\alpha \in \mathcal{A}^{N} \mid \text { for every } T \in 2^{N} \backslash\{\emptyset\}:[\{i, j\} \subseteq T \text { and }(j, i) \in D] \Rightarrow \alpha(T) \neq j\right\},
$$

We obtain the following definition.

Definition 5.4 The Restricted Selectope of game with ordered players $(v, D) \in \mathcal{G}^{N} \times \mathcal{D}^{N}$ is the set $\mathcal{S}(v, D)=\operatorname{Conv}\left(\left\{s^{\alpha}(v) \mid \alpha \in \mathcal{A}_{D}^{+N}, \Delta_{v}(T)>0\right\} \cup\left\{s^{\alpha}(v) \mid \alpha \in \mathcal{A}_{D}^{-N}, \Delta_{v}(T)<0\right\}\right)$.

We refer to the solution that assigns to every game with ordered players its Restricted Selectope as the Restricted Selectope solution. Obviously, for every $(v, D) \in$ $\mathcal{G}^{N} \times \mathcal{D}^{N}$ we have $\mathcal{S}(v, D) \subseteq S(v)$ since $\mathcal{A}_{D}^{+N} \cup \mathcal{A}_{D}^{-N} \subseteq \mathcal{A}^{N}$. Note that $\mathcal{A}_{D}^{+N}=\mathcal{A}_{D}^{-N}=\mathcal{A}^{N}$ if and only if $D=\emptyset$. Therefore $\mathcal{S}(v, D)=H(v)=\mathcal{H}(v, D)$ if $D=\emptyset$. On the other hand, $\mathcal{A}_{D}^{+N}=\mathcal{A}_{D}^{-N}=\emptyset$ if (and only if) $D$ contains a cycle. This implies that $\mathcal{S}(v, D)=\emptyset$ if there is a cycle in $D$. It is straightforwardly to verify that the Restricted Selectope solution 
satisfies efficiency, the null player property, disjoint additivity, sign preservation and the inferior player property. Hence, according to Corollary 4.6 it holds that

$$
\mathcal{S}(v, D) \subseteq \mathcal{H}(v, D)
$$

As we showed, this inclusion holds with equality when $D=\emptyset$ (since then both sets are equal to $H(v))$, whereas $\mathcal{S}(v, D)=\emptyset$ when $D$ contains a cycle. So, when we require that a solution is nonempty, we have to restrict ourselves to the class of acyclic digraphs. In a subsequent paper we intend to provide a full characterization of the restricted Selectope on the class of ordered games with acyclic digraphs. Finally, the Selectope analogue of the WIP Restricted Harsanyi set solution is given by $\mathcal{S}^{W}(v, D)=\operatorname{Conv}\left(\left\{s^{\alpha}(v) \mid \alpha \in \mathcal{A}_{D}^{+N}\right\}\right)$.

Example 5.5 Consider the game with ordered players $(v, D)$ on $N=\{1,2,3\}$ of Example 3.6.(i), i.e. $v=u_{\{1,2\}}+u_{\{1,3\}}$ and $D=\{(1,3),(2,3)\}$. The unrestricted and restricted Selectope, respectively, are given by $S(v)=H(v)=\operatorname{Conv}\left(\left\{(2,0,0)^{\top},(1,0,1)^{\top},(1,1,0)^{\top},(0,1,1)^{\top}\right\}\right)$ and $\mathcal{S}(v, D)=\operatorname{Conv}\left(\left\{(2,0,0)^{\top},(1,1,0)^{\top}\right\}\right)$.

\section{An application: airport games}

Suppose there are $n$ airplanes that want to use the same landing strip. The $n$ airplanes are different and therefore need landing strips of different size. Assume that the airplanes are labeled so that the cost of building a landing strip for airplane $i \in\{1, \ldots, n\}$ is given by $c_{i}$ satisfying $c_{1} \geq c_{2} \geq \ldots \geq c_{n} \geq 0$. The corresponding airport game (see Littlechild and Owen (1973)) is a cost game $(N, v)$ with the set of airplanes as the set of players and the characteristic function given by $v(S)=\max _{i \in S} c_{i}$ for all $S \subseteq N$. As noticed by Brânzei et. al. (2002) the dual ${ }^{6}$ airport game $v^{*}$ is given by $v^{*}=\sum_{i=1}^{n}\left(c_{i}-c_{i+1}\right) u_{[1, i]}$, with $c_{n+1}=0$, where $[1, i]$ denotes the set $\{1, \ldots, i\}$ of consecutive players including player 1. The dividend of coalition $\{1, \ldots, i\}$ is the additional cost $\left(c_{i}-c_{i+1}\right)$ of extending the landing strip that is sufficient for all aircrafts that are smaller than $i$, so that also $i$ can use it. All other coalitions have dividend equal to zero. Since eventually also all larger airplanes $j \in\{1, \ldots, i-1\}$ will use this part of the landing strip, and all smaller airplanes $j \in\{i+1, \ldots, n\}$ will not use it, it seems reasonable to divide the cost $\left(c_{i}-c_{i+1}\right)$ among $i$ and all larger airplanes $1, \ldots, i-1$. This is done by the Harsanyi set of the dual (airport) game. In addition, the Restricted Harsanyi set corresponding to the directed line-graph $L=\{(i, i+1) \in N \times N \mid i=1, \ldots, n-1\}$ (i.e. the agents are linearly ordered from 1 to $n$ ), distributes these costs in such a way that for every part of the landing strip larger airplanes pay at least as much as smaller airplanes. (Note that the WIP Restricted Harsanyi set is

\footnotetext{
${ }^{6}$ For any game $v \in \mathcal{G}^{N}$, the dual game is given by $v^{*}(S)=v(N)-v(N \backslash S)$ for all $S \subseteq N$.
} 
the same since all dividends are nonnegative.) Extreme points are given by the Shapley value in which all airplanes that use part of the landing strip pay the same share in the corresponding cost, and the unique element of the Restricted Selectope which charges all the cost for the full landing strip to the largest airplane. The Restricted Harsanyi set provides a collection of reasonable cost allocations with the Shapley value and the unique element of the Restricted Selectope as two of its extreme points.

Example 6.1 Take $N=\{1,2,3\}$ and costs $\left(c_{1}, c_{2}, c_{3}\right)^{\top}$ given by $(3,2,1)^{\top}$. The dual game of the corresponding airport game is the game $v^{*}$ on $N$ given by $v^{*}=u_{\{1\}}+u_{\{1,2\}}+u_{\{1,2,3\}}$. The Harsanyi set of this game (which by totally positiveness equals its Core) is equal to

$$
H\left(v^{*}\right)=\operatorname{Conv}\left(\left\{(3,0,0)^{\top},(2,0,1)^{\top},(1,2,0)^{\top},(1,1,1)^{\top}\right\}\right),
$$

while the Restricted Harsanyi set of this game is equal to the sum of $\mathcal{H}\left(u_{\{1\}}, L\right)=$ $\operatorname{Conv}\left(\left\{(1,0,0)^{\top}\right\}\right), \mathcal{H}\left(u_{\{1,2\}}, L\right)=\operatorname{Conv}\left(\left\{(1,0,0)^{\top},\left(\frac{1}{2}, \frac{1}{2}, 0\right)^{\top}\right\}\right)$ and $\mathcal{H}\left(u_{\{1,2,3\}}, L\right)=\operatorname{Conv}\left(\left\{(1,0,0)^{\top},\left(\frac{1}{2}, \frac{1}{2}, 0\right)^{\top},\left(\frac{1}{3}, \frac{1}{3}, \frac{1}{3}\right)^{\top}\right\}\right)$, and is thus given by

$$
\mathcal{H}\left(v^{*}, L\right)=\operatorname{Conv}\left(\left\{(3,0,0)^{\top},(2,1,0)^{\top},\left(1 \frac{5}{6}, \frac{5}{6}, \frac{1}{3}\right)^{\top},\left(2 \frac{1}{3}, \frac{1}{3}, \frac{1}{3}\right)^{\top}\right\}\right),
$$

see Figure 3. The Shapley value $S h\left(v^{*}\right)=\left(1 \frac{5}{6}, \frac{5}{6}, \frac{1}{3}\right)^{\top}$ and the unique element $s\left(v^{*}\right)=$ $(3,0,0)^{\top}$ of the Restricted Selectope element are two of its extreme points.

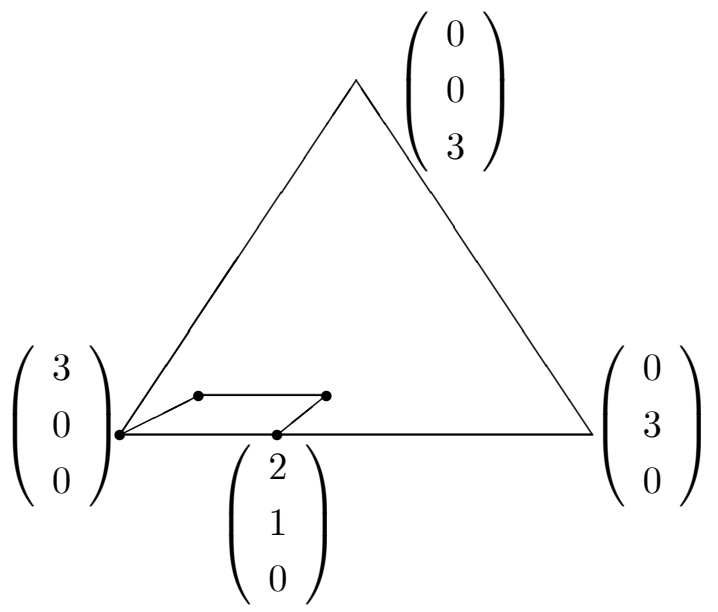

Figure 2: The Restricted Harsanyi set $\mathcal{H}\left(v^{*}, D\right)$ of Example 6.1.

Other applications where the Restricted Harsanyi solution could be applied are, for instance, sequencing situations, see e.g. Curiel et. al. (1989), auction games, see e.g. Graham et.al. (1990), and water distributions problems, see Ambec and Sprumont (2002). In van den Brink et. al. (2006) these problems are modelled as line-graph games. Considering other grph structures we can generalize these models. For example, the water 
distribution problem considers the distribution of water among agents allocated along a river from upstream to downstream. Situations with side rivers joining the main stream and river delta situations can be modelled by more general directed graphs satisfying that a pair of agents $(i, j) \in D$ if and only if $j$ is a downstream 'neighbour' of $i$.

\section{Final remarks}

In this paper we assumed the players in a cooperative TU-game to belong to a hierarchical structure which restricts the payoff distribution in the game and we propose the concept of the Restricted Harsanyi set as solution concept for such situations. This restricted Harsanyi set provides a collection of payoff distributions obtained by taking into account the hierarchical structure when distributing the dividends. Theorem 3.2 states that the Restricted Harsanyi set is a subset of the Harsanyi set of the game (without ordered players) which always contains the Shapley value of this game. Also, we have seen, for instance in Example 6.1, that the Restricted Harsanyi set cannot be obtained as the core of some modified game. This in contrast with the Harsanyi set for TU-games which equals the core of the game $v_{H}$ as defined in Section 2. For future research this opens the question whether it could be possible to define the Restricted Harsanyi set as a set of payoff vectors satisfying a system of linear (in)-equalities.

Another game theoretic model in which players in a TU-game belong to a hierarchical structure are the games with a permission structure as considered in Gilles et. al. (1992), Gilles and Owen (1994), van den Brink and Gilles (1996) and van den Brink (1997) ${ }^{7}$. In these games it is assumed that players need permission from other players before they are allowed to cooperate within a coalition. So, instead of restricting payoff distributions, in these games with permission structure the cooperation possibilities are restricted ${ }^{8}$. For instance, in the conjunctive approach to these games as developed in Gilles et. al. (1992) and van den Brink and Gilles (1996), it is assumed that each player needs permission from all its predecessors before it is allowed to cooperate. This implies that a coalition is only feasible if it is comprehensive from above. Alternatively, according to the disjunctive ap-

\footnotetext{
${ }^{7}$ Related is also the model of Faigle and Kern (1992) who consider feasible rankings of the players.

${ }^{8}$ In this sense these models fall within the theory on restricted cooperation, together with, for example, the games with limited communication (graph) structure in which the edges of an undirected graph on the set of players represent binary communication links between the players such that players can cooperate only if they are connected (see, e.g. Myerson (1977), Kalai et. al. (1978), Owen (1986) and Borm et. al. (1992)), and the games in a-prioiri coalition structure in which it is assumed that the set of players is partitioned into disjoint sets which represent social groups such that for a particular player it is more easy to cooperate with players in its own group than to cooperate with players in other groups (see, e.g., Aumann and Drèze (1974), Owen (1977), Hart and Kurz (1983) and Winter (1989)).
} 
proach a player needs permission to cooperate of at least one of its predecessors. Given the sets of feasible coalitions, a restricted game is defined which assigns to every coalition the worth of its largest feasible subset. Applying well-known solutions as the Shapley value, Core or Harsanyi set to such restricted games yields solutions for games with a permission structure. We want to stress that the two approaches of restricting payoff distributions as done in the underlying paper, and restricting cooperation possibilities as in games with a permission structure are essentially different. The Restricted Harsanyi set is a subset of the Harsanyi set of the game (without ordered players) and therefore refines the concept of Harsanyi set. In the alternative approach of restricting the cooperation possibilities, not all coalitions are feasible anymore and therefore the Core of the restricted game is larger than the Core of the original game. So, when considering, for example, the class of almost positive games (on which the Harsanyi set and Core are equal), putting restrictions on the distributions of the dividends always gives a refinement of the set of outcomes, while restricted cooperation may enlarge the set of outcomes according to these solutions.

In Section 5 we discussed shortly two alternative solutions for games with ordered players. As another alternative, we can use the concept of top cycle. A set of nodes is a top cycle, if there is a directed path between any pair of nodes within the set and there is no directed path from a node outside the set to a node in the set. A node is a top-node if it is in a top-cycle. Now the inequalities for the Restricted Harsanyi set can be weakened by requiring that top-nodes within the digraph (respectively inverse digraph) restricted to $T$ get at least (most) as much of the dividend of coalition $T$ as the players that do not belong to the top of $T$. This 'Top-node' Restricted Harsanyi set also always contains the Shapley value and is a subset of the Harsanyi set of the unrestricted game. It equals the Harsanyi set of the unrestricted game if the digraph is empty or complete. However, it never consists of a unique element and therefore does not generalize the Shapley value. So, this top-node approach does not look so attractive as alternative for the Restricted Harsanyi set. However, for the Restricted Selectope of a game with ordered players it has nice properties. In Section 5 we saw that this set is empty whenever the underlying digraph has a (directed) cycle. Weakening our selector requirement by saying that the dividend of a coalition $T$ should always be assigned to a player in the top of the digraph restricted to that coalition $T$, respectively to the top of the inverse digraph in case of negative dividends, it follows that the Restricted Selectope according to this selection rule is always nonempty since any digraph has at least one top-node. 


\section{References}

Ambec, S., and Y. Sprumont (2002), Sharing a river, Journal of Economic Theory 107, 453-462.

Aumann R.J., and J.H. Drèze (1974), Cooperative games with coalition structure, International Journal of Game Theory 3, 217-237.

Bondareva, O. (1962), The theory of the core in an n-person game, Vestnik Leningrad. Univ. 13, 141-142 (in Russian).

Borm, P., Owen, G., and Tijs, S. (1992), On the position value for communication situations, SIAM Journal of Discrete Mathematics 5, 305-320.

Brânzei, R., Fragnelli, V., and Tijs, S. (2002), Tree connected line graph peer group situations and line graph peer group games, Mathematical Methods of Operations Research 55, pp 93-106.

Brink, R. van den (1997), An axiomatization of the disjunctive permission value for games with a permission structure, International Journal of Game Theory 26, 27-43.

Brink, R. van den, and Gilles, R. P. (1996), Axiomatizations of the conjunctive permission value for games with permission structures, Games and Economic Behavior 12, 113126.

Brink, R. van den, G. van der Laan, and V.A. Vasil'ev (2006), Component efficient solutions in line-graph games with applications, forthcoming in Economic Theory, DOI 10.1007/s00199-006-0139-x.

Brink, R. van den, G. van der Laan, and V.A. Vasil'ev (2004), On the extreme points of two polytopes associated with a digraph and applications to cooperative games, Discussion Paper TI 04-069/1, Tinbergen Institute, Amsterdam/Rotterdam, The Netherlands.

Curiel, I., G. Pederzoli, and S. Tijs, Sequencing games, European Journal of Operational Research 40, (1989) 344-351.

Derks, J., H. Haller, and H. Peters (2000), The selectope for cooperative TU-games, International Journal of Game Theory 29, 23-38.

Faigle, U., and Kern, W. (1993), The Shapley value for cooperative games under precedence constraints, International Journal of Game Theory 21, 249-266. 
Gilles, R. P., and Owen, G. (1994), Cooperative games and disjunctive permission structures, Department of Economics, Virginia Polytechnic Institute and State University, Blacksburg, Virginia.

Gilles, R. P., Owen, G., and Brink, R. van den (1992), Games with permission structures: the conjunctive approach, International Journal of Game Theory 20, 277-293.

Gillies, D.B. (1953), Some Theorems on n-Person Games, Princeton University Press, Princeton, NJ.

Graham, D.A., R.C. Marshall, and J-F. Richard (1990), "Differential Payments within a Bidder Coalition and the Shapley Value", American Economic Review, 80, 493-510. Journal of Economic Theory, 38, 101-117.

Hammer, P.L., U.N. Peled, and S. Sorensen (1977), Pseudo-Boolean functions and game theory. I. Core Elements and Shapley Value, Cahiers du CERO 19, 159-176.

Harsanyi, J.C. (1959), A bargaining model for cooperative n-person games, in: Contributions to the Theory of Games IV (eds. Tucker A.W., and R.D. Luce), Princeton UP, Princeton, 325-355.

Hart S., and M. Kurz (1983), Endogeneous Formation of Coalitions, Econometrica 51, 1047-1064.

Ichiishi, T. (1981), Super modularity: applications to convex games and to the greedy algorithm for LP, Journal of Economic Theory 25, 283-286.

Kalai E., A. Postlewaite, and J. Roberts (1978), Barriers to trade and disadvantageous middlemen: nonmonotonicity of the core, Journal of Economic Theory 19, 200-209.

Littlechild, S.C, and G. Owen (1973), A simple expression for the Shapley value in a special case, Management Science 20, 370-372.

Journal of Economic Theory, 109, 90-103.

Myerson, R. B. (1977), Graphs and cooperation in games, Mathematics of Operations Research 2, 225-229.

Owen, G. (1977), Values of games with a priori unions, in: Essays in Mathematical Economics and Game Theory (eds. Henn R., and O. Moeschlin), Springer Verlag, Berlin, 76-88.

Owen, G. (1986), Values of graph-restricted games, SIAM Journal on Algebraic and Discrete Methods 7, 210-220. 
Shapley, L. S. (1953), A value for N-person games, in: Contributions to the Theory of Games, Vol II (eds. H. W. Kuhn, and A. W. Tucker), Princeton University Press, Princeton, 307-317.

Shapley, L.S. (1967), On balanced sets and cores, Naval Research Logistics Quarterly 14, 453-460.

Shapley, L.S. (1971), Cores of convex games, International Journal of Game Theory 1, $11-26$.

Vasil'ev, V.A. (1978), Support function of the core of a convex game, Optimizacija Vyp 21, 30-35 (in Russian).

Vasil'ev, V.A. (1981), On a class of imputations in cooperative games, Soviet. Math. Dokl. 23, 53-57.

Vasil'ev, V.A., and G. van der Laan, (2002), The Harsanyi set for cooperative TU-games, Siberian Advances in Mathematics 12, 97-125.

Winter, E. (1989), A value for cooperative games with levels structure of cooperation, International Journal of Game Theory 18, 227-240. 